\title{
Impacto de estratégias combinatórias no precondicionador paralelo híbrido SPIKE
}

\author{
Brenno A. Lugon ${ }^{1}$, Lucia Catabriga ${ }^{1}$, Maria C. Rangel ${ }^{1}$, Leonardo M. de Lima ${ }^{2}$ \\ ${ }^{1}$ Departamento de Informática - Universidade Federal do Espírito Santo (UFES) \\ Goiabeiras — Vitória/ES - Brasil - CEP 29075-910 \\ ${ }^{2}$ Instituto Federal do Espírito Santo (IFES) \\ \{blugon, luciac, crangel\}@inf.ufes.br, lmuniz@ifes.edu.br
}

\begin{abstract}
In this paper, we use the parallel hybrid SPIKE algorithm as a preconditioner for a nonstationary iterative method combining distributed and shared memory architectures, MPI and OpenMP. In order to obtain a good preconditioner we solve a set of combinatorial problems such as reorderings and graph partitioning. We present the results evaluating the influence of each strategy on the convergence and CPU time of the iterative solver.
\end{abstract}

Resumo. Neste trabalho, utilizamos o algoritmo paralelo híbrido SPIKE como um precondicionador para um método iterativo não-estacionário combinando as arquiteturas de memória distribuída e compartilhada, MPI e OpenMP. A fim de obter um bom precondicionador, resolvemos um conjunto de problemas combinatórios como reordenamentos e particionamento de grafos. Apresentamos os resultados avaliando a influência de cada estratégia na convergência e tempo de CPU do método iterativo.

\section{Introdução}

Para a resolução de sistemas lineares de grande porte, é interessante a utilização de métodos iterativos baseados em projeções de subespaços de Krylov por suas boas propriedades numéricas e computacionais. Além disso, o uso de precondicionadores acelera a convergência e, consequentemente, melhora a eficiência desses métodos. na obtenção da solução. Em aplicações paralelas, o algoritmo híbrido SPIKE [Polizzi and Sameh 2006, Polizzi and Sameh 2007], criado inicialmente para resolver um sistema linear, pode também ser usado como precondicionador para o método iterativo. Ele é dito um método híbrido por ser capaz de tirar vantagem da robustez dos métodos diretos e do baixo custo computacional dos métodos iterativos.

Recentemente o uso de matchings para scalings e reordenamento de matrizes demonstraram ser poderosas técnicas no processo de solução de sistemas lineares esparsos. Em [Hogg and Scott 2015], o algoritmo de escalonamento MC 64 aplicado a problemas com matrizes simétricas indefinidas, amplamente utilizado, é particularmente eficaz em comparação com outros scalings e técnicas testadas.

Neste trabalho iremos examinar os impactos de técnicas de otimização na convergência e tempo de execução do método iterativo GMRES precondicionado com o algoritmo híbrido baseado no SPIKE. As etapas que envolvem obter soluções por métodos diretos foram computadas utilizando o software PARDISO ${ }^{1}$, em uma abordagem conhecida como PSPIKE (PARDISO + SPIKE) [Manguoglu et al. 2009].

\footnotetext{
${ }^{1} \mathrm{http} / / /$ www.pardiso-project.org/
} 


\section{SPIKE clássico}

O algoritmo paralelo híbrido SPIKE tem como objetivo encontrar a solução de um sistema linear da forma $A x=f$ usando computação paralela, sendo $A=\left\{a_{i j}\right\}_{n \times n}$ uma matriz esparsa diagonal dominante. Podemos dividir o algoritmo SPIKE em três etapas principais: pré-processamento, fatoração e pós-processamento [Polizzi and Sameh 2006].

\subsection{Pré-processamento}

A primeira etapa do pré-processamento consiste em transformar uma matriz esparsa geral em uma matriz com estrutura de banda, i.e., com os elementos não nulos dispostos próximos da diagonal principal e largura de banda relativamente pequena. Esta transformação é uma condição necessária para que o método SPIKE possa ser aplicado e este objetivo pode ser alcançado através de um reordenamento, detalhado na Seção 4.2. Em seguida, é necessário particionar a matriz com estrutura de banda em uma matriz bloco tridiagonal, a fim de se obter os blocos $C_{i}, A_{i}$ e $B_{i}$, como mostra a Figura 1 . Este problema pode ser interpretado como um problema combinatório de particionamento de grafos que será explicado na Seção 4.3.

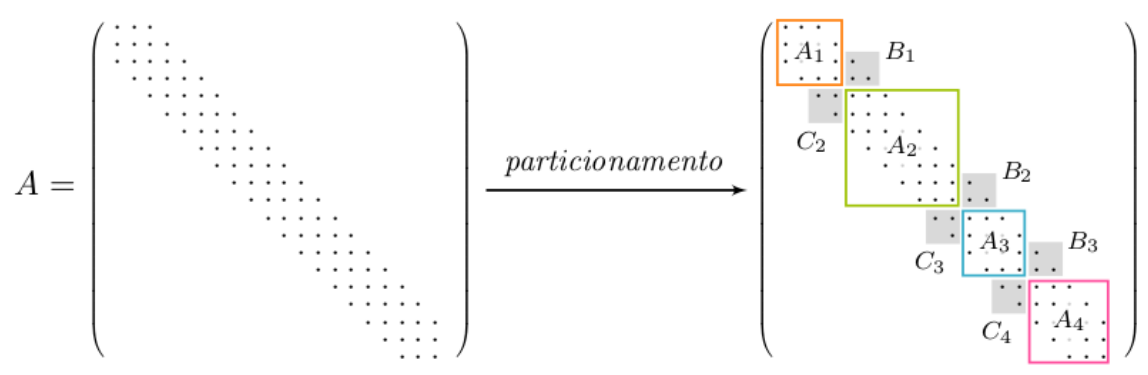

Figura 1. Particionamento bloco tridiagonal em 4 processadores

\subsection{Fatoração}

Diferente das fatorações usuais muito usadas pelos métodos diretos como $A=\mathbf{L U}$ ou $A=\mathbf{L D L}^{\mathbf{T}}$, o algoritmo SPIKE se baseia na fatoração $A=D S$, onde $D$ é uma matriz bloco diagonal e $S$ é chamada de matriz de spikes. A Figura 2 mostra um exemplo dessa fatoração para um particionamento em 4 processadores.

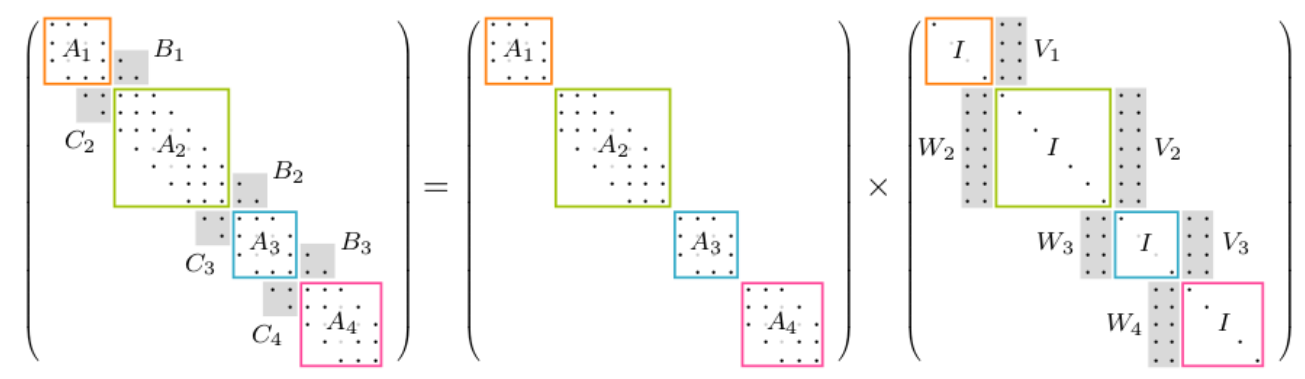

Figura 2. Decomposição $A=D S$

A matriz $A$ é formada pelas matrizes bloco diagonais $A_{i}(i=1, \ldots, p)$ e pelas matrizes bloco de acoplamento $B_{i}(i=1, \ldots, p-1)$ e $C_{i}(i=2, \ldots, p)$. Para garantir que todos os elementos não nulos dispostos fora das matrizes bloco diagonais sejam cobertos pelas matrizes bloco de acoplamento, $B_{i}$ e $C_{i}$ possuem dimensão $\mathbf{k} \times \mathbf{k}$, onde $\mathbf{k}$ é a 
largura de banda de $A$. A matriz $D$ é formada pelas matrizes bloco diagonais quadradas $A_{i}$ e, assumindo a não singularidade de cada bloco $A_{i}$, a matriz $S=D^{-1} A$ é formada pelas matrizes spikes $V_{i}(i=1, \ldots, p-1)$ e $W_{i}(i=2, \ldots, p)$, densas e de dimensão $n_{i} \times \mathbf{k}$, sendo $n_{i}$ o número de linhas dos blocos $A_{i}$.

As matrizes spikes $V_{i}$ e $W_{i}$ são dadas por

$$
V_{i}=A_{i}^{-1}\left(\begin{array}{c}
0 \\
B_{i}
\end{array}\right) \text { e } W_{i}=A_{i}^{-1}\left(\begin{array}{c}
C_{i} \\
0
\end{array}\right)
$$

e, em cada processador $i$, podem ser calculadas resolvendo os sistemas

$$
A_{i} V_{i}=\left(\begin{array}{c}
0 \\
B_{i}
\end{array}\right) \text { e } A_{i} W_{i}=\left(\begin{array}{c}
C_{i} \\
0
\end{array}\right)
$$

onde o primeiro processador calcula apenas $V_{1}$ e o último processador $p$ calcula apenas $W_{p}$. Os sistemas da Equação (2) possuem múltiplos vetores de termos independentes e, consequentemente, terão múltiplos vetores como solução. Estes sistemas serão resolvidos via método direto de fatoração $\mathbf{L} \mathbf{U}, \operatorname{com} A_{i}=\mathbf{L}_{i} \mathbf{U}_{i}$, e podem ser representados por

$$
\mathbf{L}_{i} \mathbf{U}_{i}\left[V_{i}, W_{i}\right]=\left[\left(\begin{array}{c}
0 \\
B_{i}
\end{array}\right),\left(\begin{array}{c}
C_{i} \\
0
\end{array}\right)\right] .
$$

\subsection{Pós-processamento}

O sistema $A x=f$ com a fatoração $A=D S$ deve ser resolvido em duas etapas:

1. resolver $D g=f$

2. resolver $S x=g$

O sistema $D g=f$ é totalmente desacoplado, ou seja, pode ser resolvido em paralelo sem gastos com comunicação e sincronização entre os processadores. Isso porque a matriz $D$ é formada apenas pelas matrizes bloco diagonais $A_{i}$, independentes entre si. Portanto, podemos resolver esta etapa usando a fatoração LU, i.e., em cada processador $i$, resolver o sistema $\mathbf{L}_{i} \mathbf{U}_{i} g=f$.

O segundo sistema, $S x=g$, é fracamente acoplado, ou seja, pode ser resolvido com pouca comunicação entre processadores. Podemos observar que resolver este sistema é equivalente a resolver um sistema $\hat{S} \hat{x}=\hat{g}$ de menor complexidade, chamado de sistema reduzido. Para resolvê-lo, considere o particionamento de $V_{i}, W_{i}$ e $x_{i}, g_{i}$ como

$$
V_{i}=\left(\begin{array}{c}
V_{i}^{(t)} \\
V_{i}^{(m)} \\
V_{i}^{(b)}
\end{array}\right), W_{i}=\left(\begin{array}{c}
W_{i}^{(t)} \\
W_{i}^{(m)} \\
W_{i}^{(b)}
\end{array}\right) \quad \text { e } \quad x_{i}=\left(\begin{array}{c}
x_{i}^{(t)} \\
x_{i}^{(m)} \\
x_{i}^{(b)}
\end{array}\right), g_{i}=\left(\begin{array}{c}
g_{i}^{(t)} \\
g_{i}^{(m)} \\
g_{i}^{(b)}
\end{array}\right),
$$

onde $V_{i}^{(t)}, V_{i}^{(b)}, W_{i}^{(t)}$ e $W_{i}^{(b)}$ são as extremidades das matrizes spikes $V_{i}$ e $W_{i}$, de tamanho $n_{i} \times \mathbf{k}$. Da mesma forma, $x_{i}^{(t)}, x_{i}^{(b)}$ e $g_{i}^{(t)}, g_{i}^{(b)}$ representam as extremidades dos vetores $x_{i}$ e $g_{i}$ de tamanho k. Os sobrescritos $(t),(m)$, e $(b)$ são abreviações de top (cima), middle (meio) e bottom (baixo), respectivamente. A partir destas definições, o sistema reduzido $\hat{S} \hat{x}=\hat{g}$ pode ser representado por $p-1$ blocos diagonais, onde o $i$-ésimo bloco, seus blocos correspondentes fora da diagonal, o vetor de solução e o vetor de termos independentes são dados, respectivamente, por

$$
\left(\begin{array}{cc}
I & V_{i}^{(b)} \\
W_{i+1}^{(t)} & I
\end{array}\right),\left(\begin{array}{cc}
W_{i}^{(b)} & 0 \\
0 & 0
\end{array}\right),\left(\begin{array}{cc}
0 & 0 \\
0 & V_{i+1}^{(t)}
\end{array}\right),\left(\begin{array}{c}
\hat{x}_{i}^{(b)} \\
\hat{x}_{i+1}^{(t)}
\end{array}\right) \text { e }\left(\begin{array}{c}
\hat{g}_{i}^{(b)} \\
\hat{g}_{i+1}^{(t)}
\end{array}\right)
$$


Uma vez obtida a solução $\hat{x}$ do sistema reduzido, representado em (5), a solução $x$ do sistema global pode ser obtida com perfeito paralelismo fazendo

$$
\left\{\begin{array}{l}
x_{1}=g_{1}-V_{1} \hat{x}_{2}^{(t)} \\
x_{i}=g_{i}-W_{i} \hat{x}_{i-1}^{(b)}-V_{i} \hat{x}_{i+1}^{(t)} \quad i=2, \ldots, p-1 \\
x_{p}=g_{p}-W_{p} \hat{x}_{p-1}^{(b)}
\end{array} .\right.
$$

Entretanto, utilizando a abordagem descrita, a etapa de montagem do sistema reduzido $\hat{S} \hat{x}=\hat{g}$ pode se tornar muito custosa computacionalmente. Isso porque as matrizes $V_{i}$ e $W_{i}$ são densas com quantidade de elementos igual a $n_{i} \times \mathbf{k}$ para cada $i$, o que demanda uma grande quantidade de memória para armazenamento. Com o objetivo de melhorar a eficiência do algoritmo, reduzindo os cálculos computacionais envolvidos na decomposição LU e o uso de memória - sem comprometer a exatidão, a menos de erros de arredondamento -, podemos calcular apenas as extremidades superiores e inferiores de tamanho $\mathbf{k} \times \mathbf{k}$ das matrizes $V_{i}, W_{i}$ e do vetor $g_{i}$. Assim, a solução final do sistema $A x=f$ pode ser recuperada fazendo

$$
\left\{\begin{array}{l}
\mathbf{L}_{1} \mathbf{U}_{1} x_{1}=f_{1}-B_{1} \hat{x}_{2}^{(t)} \\
\mathbf{L}_{i} \mathbf{U}_{i} x_{i}=f_{i}-C_{i} \hat{x}_{i-1}^{(b)}-B_{i} \hat{x}_{i+1}^{(t)} \quad i=2, \ldots, p-1 \\
\mathbf{L}_{p} \mathbf{U}_{p} x_{p}=f_{p}-C_{p} \hat{x}_{p-1}^{(b)}
\end{array} .\right.
$$

\section{SPIKE como precondicionador}

O algoritmo SPIKE atuando como precondicionador, também chamado de SPIKE-TU ou SPIKE Truncado, apresenta mudanças, em relação ao SPIKE clássico, essenciais para aprimorar o paralelismo e diminuir o número de operações de ponto flutuante.

A primeira mudança do algoritmo SPIKE, atuando como precondicionador, está na obtenção das matrizes spikes $V_{i}$ e $W_{i}$. Considerando que as matrizes bloco diagonais $A_{i}$ sejam diagonais dominantes, podemos usar somente os blocos inferiores, de dimensão $\mathbf{k} \times \mathbf{k}$, das matrizes $\mathbf{L}$ e $\mathbf{U}$ da fatoração $\mathbf{L} \mathbf{U}$ para obter $V_{i}$ e, analogamente, os blocos superiores, de dimensão $\mathbf{k} \times \mathbf{k}$, de $\mathbf{U}$ e $\mathbf{L}$ da fatoração $\mathbf{U L}$ para obter $W_{i}$. Apesar de essa estratégia necessitar de mais uma fatoração, a UL, [Polizzi and Sameh 2006] afirmam, baseados em seus experimentos, que essa estratégia é mais eficiente do que usar apenas a fatoração $\mathbf{L} \mathbf{U}$, pois assim seria necessário calcular a matriz de spikes $W_{i}$ total em cada bloco diagonal.

O próxima mudança no SPIKE-TU é com relação ao sistema reduzido. Como o algoritmo SPIKE-TU será usado como um precondicionador, não há necessidade de se obter solução exata desse sistema, portanto, uma solução aproximada e mais simples de construir é suficiente. Neste caso, resolver o sistema reduzido truncado diminui a quantidade de comunicação e tem pouca influência na convergência do método iterativo. Sendo assim, o sistema reduzido truncado $\tilde{S}_{i}$ do processador $i$ é formado somente pelas matrizes spikes $V_{i}^{(b)}$ e $W_{i+1}^{(t)}$ e pode ser representado matricialmente como

$$
\tilde{S}_{i}=\left(\begin{array}{cc}
I & V_{i}^{(b)} \\
W_{i+1}^{(t)} & I
\end{array}\right) .
$$

Como podemos observar, os blocos $W_{i}^{(b)}$ e $V_{i+1}^{(t)}$ mostrados em (5) são desconsiderados. Portanto, sendo $p$ a quantidade total de processadores, o sistema 
reduzido truncado $\tilde{S}=\operatorname{diag}\left\{\tilde{S}_{1}, \tilde{S}_{2}, \ldots, \tilde{S}_{p-1}\right\}$ será resolvido por partes em cada processador. Como o este sistema no processador $i$ é formado pelas matrizes spikes $V_{i}^{(b)}$ e $W_{i+1}^{(t)}$, é necessário que todo processador $i+1$ envie a matriz $W_{i+1}^{(t)}$ para o processador $i$ que deve montar e resolver sua respectiva parte do sistema.

Para criar a matriz precondicionadora $M$, é conveniente aplicar um conjunto de reordenamentos na matriz $A$ de forma que os blocos diagonais $A_{i}$ e as matrizes bloco de acoplamento $B_{i}$ e $C_{i}$ contenham os elementos mais significativos da matriz. Sendo assim, a matriz $M$ é criada considerando os seguintes reordenamentos

$$
M=K Q P D_{r} A D_{c} P^{T} K^{T},
$$

sendo $D_{r}$ e $D_{c}$ fatores de scaling, $P$ uma permutação simétrica com o objetivo de reduzir a largura de banda da matriz, $Q$ uma permutação não simétrica para mover os maiores elementos para a diagonal e $K$ uma permutação simétrica que move os elementos mais significativos para os blocos de acoplamento.

Esses reordenamentos (ou permutações) são modelados por problemas combinatórios, resumidos na próxima seção.

\section{Estratégias combinatórias}

Cada transformação aplicada na matriz original pode ser abordada como problemas combinatórios em grafos, a saber: matching perfeito em grafos, particionamento de grafos e rerrotulação de vértices de um grafo. Dentre os objetivos que desejamos alcançar estão:

1. garantir a não-singularidade de cada bloco diagonal.

2. obter uma matriz com estrutura de banda.

3. obter as matrizes bloco diagonais e de blocos de acoplamento.

4. mover elementos mais significativos para os blocos de acoplamento.

\subsection{Matching e Scaling}

Na etapa de fatoração do algoritmo SPIKE, mostrado na Equação (2), as matrizes bloco diagonais $A_{i}$ precisam ser resolvidas via método direto usando a fatoração LU. Para isso, é necessário que esses blocos sejam não-singulares, ou seja, admitam uma inversa. Esse objetivo pode ser interpretado como um matching perfeito em grafos capaz de mover os elementos mais significativos para a diagonal principal. Portanto, seja $Q$ uma permutação de linha não simétrica do matching, o novo sistema linear resultante é da forma

$$
Q A x=Q f .
$$

Os algoritmos mais eficientes para encontrar matchings maximais em um grafo bipartido, descritos em [Duff and Koster 2000], estão presentes na biblioteca HSL, especificamente no algoritmo MC $64^{2}$. O algoritmo usado neste trabalho é chamado de matching bipartido valorado (weighted bipartite matching) e seu objetivo é encontrar um conjunto de entradas, que não estejam na mesma linha e coluna, de forma que o produto ou a soma dessas entradas sejam maximizadas.

A sub-rotina de cálculo do matching presente na biblioteca HSL MC64, adicionalmente, é capaz de calcular fatores que tornam a matriz diagonal dominante. A técnica, chamada de scaling, consiste em multiplicar a matriz por fatores de linha

\footnotetext{
${ }^{2}$ http://www.hsl.rl.ac.uk/catalogue/mc64.html
} 
e coluna de tal forma que as entradas não nulas da diagonal principal assumam valor absoluto igual a 1.0 e as demais entradas, valor absoluto menor ou igual a 1.0. Segundo [Sathe et al. 2012], o scaling tem grande influência na escalabilidade e robustez do SPIKE quando usado como precondicionador. Para aplicar o scaling, considere os logaritmos naturais dos fatores do scaling $D_{r}=\exp \left\{u_{1}, u_{2}, u_{3}, \ldots, u_{n}\right\}$ para as linhas, e $D_{c}=\exp \left\{v_{1}, v_{2}, v_{3}, \ldots, v_{n}\right\}$ para as colunas. Assim, o novo sistema após o scaling será

$$
D_{r} A D_{c}^{T} D_{c} x=D_{r} f .
$$

onde cada elemento não nulo da matriz $A$ sofre a influência $a_{i j}=a_{i j} * \exp \left(u_{i}+v_{j}\right)$.

\subsection{Reordenamento}

Para que o algoritmo SPIKE possa ser aplicado para uma matriz geral, devemos primeiramente transformá-la em uma matriz com estrutura de banda através de um reordenamento que minimize sua largura de banda. Isso porque o SPIKE necessita que todos os elementos não nulos estejam cobertos pelas matrizes bloco diagonais $A_{i}$ e pelas matrizes bloco de acoplamento, $B_{i}$ e $C_{i}$.

Neste trabalho, os algoritmos utilizados foram o Espectral, proposto por [Barnard et al. 1993] e o Espectral Valorado, proposto por [Manguoglu et al. 2010]. Os algoritmos da biblioteca HSL MC $73^{3}$ usam o conceito de conectividade algébrica da matriz, bem como o vetor de Fiedler [Fiedler 1973]. O objetivo do Espectral Valorado, além de minimizar a largura de banda da matriz, é tentar deslocar as entradas mais significativas para próximo da diagonal principal garantindo maior eficácia do precondicionador SPIKE. Ao fim da execução dessa estratégia, temos uma matriz de permutação simétrica $P$ que minimiza a largura de banda da matriz original e deve ser aplicada em todo o sistema linear de acordo com

$$
P A P^{T} P x=P f \text {. }
$$

\subsection{Particionamento}

Os principais objetivos do particionamento são reduzir a comunicação entre os processadores e garantir que cada partição tenha uma quantidade balanceada de trabalho a ser realizado, i.e., garantir que cada partição tenha aproximadamente a mesma quantidade de elementos não nulos. Como o tempo de execução de um programa paralelo é medido em função do processo mais lento, equilibrar a quantidade de elementos não nulos é assegurar que cada nó distribuído realize, aproximadamente, a mesma quantidade de operações de ponto flutuante.

Este particionamento é modelado como um problema específico, chamado de Chains-on-chains partitioning (CCP) [Pinar and Aykanat 2004], que pode ser resolvido em tempo polinomial. Neste trabalho, o algoritmo MinMax [Manne and Sørevik 1995] é empregado para obter a solução exata.

\subsection{Problema Quadrático da Mochila}

Nesta estratégia, o objetivo é aplicar uma permutação simétrica $K$ para concentrar elementos mais significativos, em módulo, dentro dos blocos de acoplamento $B_{i} \mathrm{e}$ $C_{i}$ de tamanho $\mathbf{k} \times \mathbf{k}$. Para este fim, utilizamos a heurística DeMin, proposta por [Sathe et al. 2012], que encontra uma permutação tal que o novo sistema deve ser

$$
K A K^{T} K x=K f \text {. }
$$

\footnotetext{
${ }^{3}$ http://www.hsl.rl.ac.uk/catalogue/hsl_mc73.html
} 


\section{Testes computacionais}

Os testes computacionais apresentados a seguir, utilizam uma abordagem híbrida entre MPI e OpenMP. Neste paradigma de programação, multicomputadores exploram o paralelismo usando memória distribuída enquanto que multiprocessadores garantem as vantagens do uso de memória compartilhada. A Figura 3 exibe um exemplo do esquema arquitetural dessa abordagem híbrida. Diferentes nós (Memória +4 cores) se comunicam usando MPI via rede, e os cores em cada nó se comunicam usando OpenMP.

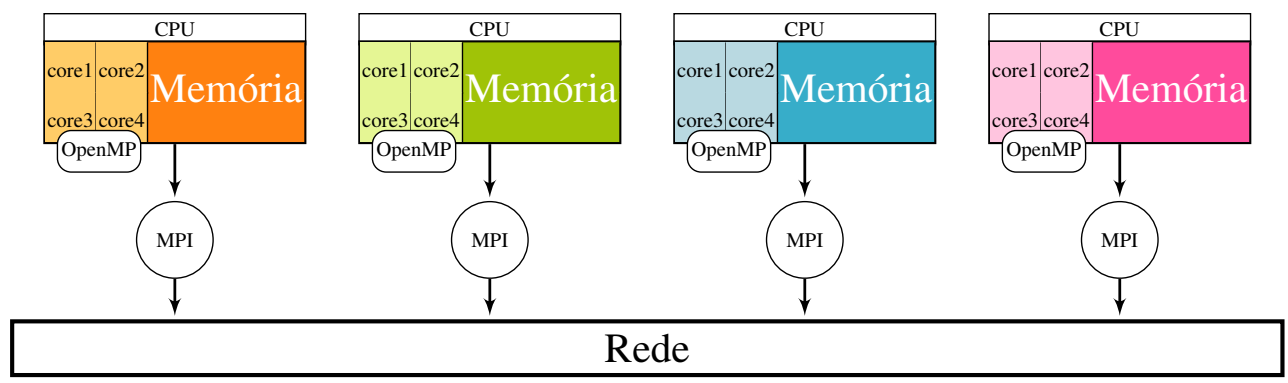

Figura 3. Abordagem híbrida MPI e OpenMP

Os experimentos apresentados nesta seção foram realizados em dois clusters: o cluster Enterprise $3^{4}$ da Universidade Federal do Espírito Santo e o cluster Altix$\mathrm{xe}^{5}$ do Laboratório Nacional de Computação Científica (LNCC) localizado no Rio de Janeiro. O primeiro é um cluster mais antigo e, portanto, possui configurações modestas comparadas com hardwares atuais. Com isso, desejamos avaliar o comportamento dos nossos experimentos neste cluster, assim como em um cluster mais potente, o Altix-xe.

O cluster Enterprise 3 conta com 24 nós Quad Core Intel 2 Q6600 (96 cores), com frequência de clock de $2.4 \mathrm{GHz}, 4 \mathrm{MB}$ de memória cache L2 e $4 \mathrm{~GB}$ de RAM, interconectados com um switch Gigabit Ethernet 48-Port 4200G 3COM. O cluster Altixxe opera com 30 unidades de processamento, cada qual com 24 GB de RAM e 2 processadores Intel Xeon E5520 2.27GHz Quad Core, totalizando 8 cores por unidade. Neste cluster, o máximo de processadores permitido por usuário é 96.

Todas as etapas do precondicionador SPIKE-TU que envolvem cálculos usando métodos diretos foram resolvidos utilizando o software PARDISO. Para os experimentos realizados neste trabalho, aplicamos o método iterativo não-estacionário GMRES. Os códigos em $\mathrm{C}$ foram compilados com GNU no cluster Enterprise 3 e com compiladores Intel no cluster Altix-xe. A Tabela 1 apresenta as principais características de um conjunto de matrizes obtidas do repositório de matrizes esparsas da Universidade da Flórida [Davis and Hu 2011]. As informações na tabela são o nome da matriz, dimensão $n$ e quantidade de elementos não nulos nnz, seu padrão de simetria e área de aplicação.

\subsection{Influência do precondicionador SPIKE}

Neste primeiro teste, realizado no cluster Altix-xe, iremos avaliar o influência do precondicionador SPIKE-TU no conjunto de matrizes da Tabela 1, com relação ao tempo computacional e número de iterações do GMRES de uma execução sem precondicionador e com o precondicionador. Foram usados quatro nós com memória distribuída e quatro

\footnotetext{
${ }^{4}$ enterprise3.lcad.inf.ufes.br/ganglia

${ }^{5} \mathrm{http}: / / \mathrm{www} . \operatorname{lncc} \cdot$ br/altix-xe
} 
Tabela 1. Características das matrizes testadas

\begin{tabular}{llrrrr}
\hline & matriz & dimensão(n) & não nulos $(\mathrm{nnz})$ & simétrica? & área de aplicação \\
\hline 1 & finan512 & 74.752 & 596.992 & sim & Otimização Financeira \\
2 & fem_3D_thermal1 & 17.880 & 430.740 & não & Elementos Finitos 3D \\
3 & fp & 7.548 & 834.222 & não & Eletromagnetismo \\
4 & rai__79841 & 79.841 & 553.921 & sim & Transferência de Calor \\
5 & mario001 & 38.434 & 204.912 & não & Redução de Modelo \\
6 & dw8192 & 8.192 & 41.746 & não & Ondas Dielétricas \\
7 & gemat12 & 4.929 & 33.111 & não & Rede de Energia \\
\hline
\end{tabular}

threads. O símbolo $\dagger$ significa que o GMRES não convergiu após 100.000 iterações. A tolerância usada para o GMRES foi $1 \times 10^{-8}$ para todas as matrizes, exceto para a matriz $\mathrm{fp}$, que foi $1 \times 10^{-14}$. O tamanho $\mathrm{k}$ dos blocos de acoplamento foi fixado em 50. A influência das estratégias combinatórias no precondicionador SPIKE são apresentadas na Tabela 2. As estratégias combinatórias são representadas por símbolos na ordem (scaling, matching, reordenamento, problema quadrático da mochila), onde os símbolos $\bullet$ e $\circ$ representam estratégias combinatórias utilizadas e não utilizadas, respectivamente. No caso do reordenamento, o símbolo • indica Espectral e •, Espectral Valorado.

Tabela 2. Influência do precondicionador SPIKE

\begin{tabular}{llrrrrr} 
& & \multicolumn{2}{c}{ sem precondicionador } & \multicolumn{2}{c}{ com precondicionador } \\
\cline { 3 - 6 } & matriz & estratégias & iterações & tempo & iterações & tempo \\
\hline 1 & finan512 & $\circ \circ \bullet \circ$ & 45 & 0,11 & 7 & 0,99 \\
2 & fem_3D_thermal1 & $\bullet \circ \bullet \circ$ & 59 & 0,04 & 20 & 0,97 \\
3 & fp & $\circ \circ \bullet \circ$ & $\dagger$ & $\dagger$ & 48 & 0,80 \\
4 & rail_79841 & $\bullet \bullet \bullet \circ$ & 42.413 & 171,90 & 163 & 2,71 \\
5 & mario001 & $\circ \circ \bullet \circ$ & 25.619 & 44,43 & 97 & 1,34 \\
6 & dw8192 & $\bullet \bullet \bullet \circ$ & $\dagger$ & $\dagger$ & 27 & 0,11 \\
7 & gemat12 & $\circ \bullet \bullet \bullet$ & $\dagger$ & $\dagger$ & 14 & 0,04 \\
\hline
\end{tabular}

Analisando os resultados da Tabela 2, vemos que o uso do precondicionador é, na maioria das vezes, muito vantajoso. Matrizes com convergência lenta, como a rail_79841 e a mario001, apresentam grande redução no número de iterações e, consequentemente, do tempo de execução. Além disso, parte das matrizes que não convergem para a solução sem o uso do precondicionador, passaram a convergir após seu uso, o que mostra a importância desta técnica. Entretanto, matrizes que convergem rapidamente não apresentaram grandes vantagens computacionais, pois o precondicionador SPIKE, apesar de reduzir o número de iterações, torna cada iteração mais custosa. Por esse motivo, o tempo de processamento para matrizes como finan512 e fem_3D_thermal1 não reduziu com relação ao método sem precondicionador. É importante dizer que nem sempre a aplicação de todas as estratégias combinatórias garantirão um melhor precondicionador. Na prática, é preciso conhecer as características do problema e testar a melhor combinação de estratégias.

\subsection{Influência das estratégias combinatórias}

Nessa seção, iremos analisar o comportamento do GMRES com o precondicionador SPIKE quando aplicado às estratégias combinatórias separadamente. Todos os testes apresentados a seguir foram executados no cluster Altix-xe. 


\subsubsection{Matching e Scaling}

O matching é usado para mover os elementos mais significativos, em módulo, para diagonal principal. Esta técnica é fundamental para matrizes que não possuem elementos na diagonal principal, pois além da dificuldade de realizar a fatoração LU, outras estratégias também não apresentam um bom comportamento. A matriz gemat 12 não possui a diagonal principal bem definida e, por isso, ela não converge (ver Tabela 2). Além disso, o reordenamento Espectral encontra dificuldades em reduzir sua largura de banda. A Figura 4 mostra a matriz gemat 12 em três estágios: (a) configuração de esparsidade original, (b) após a aplicação do matching e (c) após o reordenamento Espectral. Podemos notar que a utilização do matching antes do reordenamento possibilitou a convergência do método GMRES com precondicionador SPIKE (ver Tabela 2).

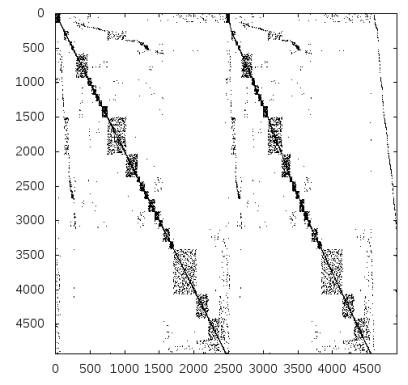

(a)

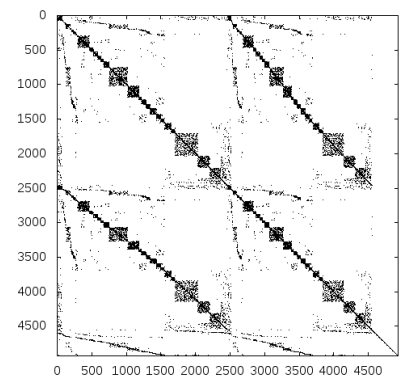

(b)

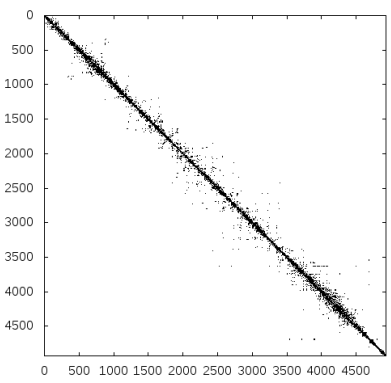

(c)

Figura 4. Influência do matching - Configuração da esparsidade

A Tabela 3 mostra a influência na construção do precondicionador SPIKE quando aplicamos o scaling na matriz rail_79841, com p representando o número de processadores distribuídos. Podemos perceber a expressiva redução do número de iterações e do tempo de processamento quando usamos o scaling. Também vale destacar que o precondicionador SPIKE, em algumas situações, pode não apresentar um bom speedup. Isso ocorre porque a medida que a quantidade de processadores aumenta, o número de iterações do método GMRES tende a crescer.

Tabela 3. Influência do scaling na matriz rail__ 79841

\begin{tabular}{lrrrr}
\hline sem scaling & $\mathbf{p = 2}$ & $\mathbf{p = 4}$ & $\mathbf{p}=\mathbf{8}$ & $\mathbf{p = 1 6}$ \\
\hline iterações & 150 & 549 & 1.644 & 4.640 \\
tempo (seg) & 5,60 & 9,34 & 15,37 & 16,16 \\
\hline com scaling & $\mathbf{p = 2}$ & $\mathbf{p = 4}$ & $\mathbf{p}=\mathbf{8}$ & $\mathbf{p = 1 6}$ \\
\hline iterações & 98 & 329 & 791 & 2.296 \\
tempo (seg) & 3,92 & 5,77 & 7,51 & 8,08 \\
\hline
\end{tabular}

\subsubsection{Espectral Valorado}

O reordenamento Espectral Valorado tem por objetivo mover os elementos mais significativos para próximo da diagonal principal, podendo exercer bastante influência na convergência do método iterativo. A Tabela 4 detalha o impacto desse reordenamento aplicado à matriz dw8192. Note que, o Espectral Valorado reduziu de forma significativa 
o número de iterações e o tempo de processamento com relação ao Espectral. Além disso, o Espectral Valorado diminuiu o tempo de processamento, em quase todas as situações, quando aumentamos o número de processadores.

Tabela 4. Influência do Espectral Valorado na matriz dw 8192

\begin{tabular}{lrrrr}
\hline Espectral & $\mathbf{p}=\mathbf{2}$ & $\mathbf{p}=\mathbf{4}$ & $\mathbf{p}=\mathbf{8}$ & $\mathbf{p}=\mathbf{1 6}$ \\
\hline iterações & 196 & 614 & 1.165 & 1.992 \\
tempo (seg) & 0,83 & 2,28 & 6,04 & 18,81 \\
\hline Espectral Valorado & $\mathbf{p}=\mathbf{2}$ & $\mathbf{p = 4}$ & $\mathbf{p}=\mathbf{8}$ & $\mathbf{p}=\mathbf{1 6}$ \\
\hline iterações & 14 & 27 & 42 & 88 \\
tempo (seg) & 0,15 & 0,11 & 0,09 & 0,10 \\
\hline
\end{tabular}

\subsubsection{Problema quadrático da mochila}

A ação de mover os elementos mais significativos para dentro dos blocos de acoplamento $B_{i}$ e $C_{i}$ pode ser muito benéfica. A Tabela 5 mostra a matriz gemat 12 onde podemos ver a redução do número de iterações e tempo de CPU após a aplicação dessa estratégia. Medimos a qualidade dessa estratégia pela quantidade de elementos movidos para os blocos. Considerando $\mathrm{p}=\mathbf{2}$ foram movidos 138 elementos e para $\mathrm{p}=4,298$ elementos.

Tabela 5. Influência do problema quadrático da mochila da matriz gemat 12

\begin{tabular}{lrr}
\hline sem problema quadrático da mochila & $\mathbf{p = 2}$ & $\mathbf{p = 4}$ \\
\hline iterações & 46 & 83 \\
tempo (seg) & 0,10 & 0,09 \\
\hline com problema quadrático da mochila & $\mathbf{p}=\mathbf{2}$ & $\mathbf{p = 4}$ \\
\hline iterações & 8 & 13 \\
tempo (seg) & 0,05 & 0,04 \\
\hline
\end{tabular}

\subsection{Desempenho das estratégias combinatórias}

Foi considerada uma matriz de grande porte $(n=1.043 .474$ e $n n z=7.294 .192)$, denotada por fem_2d_pud, oriunda de um problema bidimensional de convecçãodifusão discretizado pelo método dos elementos finitos. Este problema apresenta uma convergência lenta e, portanto, o precondicionador SPIKE utilizado é bastante eficaz.

Para garantir uma matriz com estrutura de banda, no primeiro conjunto de testes foi aplicado o reordenamento Espectral padrão. Em seguida, escolhemos a melhor configuração de estratégias combinatórias para este problema, isto é, Espectral Valorado com o scaling. Vale ressaltar que os tempos da aplicação das estratégias Espectral padrão e Espectral Valorado com scaling estão na mesma ordem de grandeza.

Considerando que todo o processo de solução fosse sequencial, o préprocessamento representaria menos de $10 \%$ do tempo para resolver o sistema linear. Contudo, nas execuções paralelas, este pré-processamento pode representar mais que o dobro do tempo para resolver este mesmo sistema. As Tabelas 6 e 7 mostram o número de iterações e o tempo de execução dessa aplicação para 2, 4, 8 e 16 processadores MPI e 1, 2 e 4 threads OpenMP, nos clusters Enterprise 3 e Altix-xe, respectivamente. Como podemos perceber nas tabelas, a aplicação das estratégias combinatórias reduziu o tempo de processamento e quantidade de iterações em ambos os clusters. 
Tabela 6. Tempos (seg.) para diferentes estratégias combinatórias - Enterprise 3

\begin{tabular}{|l|crrr|crrr|}
\cline { 2 - 9 } \multicolumn{1}{c|}{} & \multicolumn{4}{|c|}{ Espectral } & \multicolumn{4}{c|}{ Espectral Valorado + scaling } \\
\cline { 2 - 9 } \multicolumn{1}{c|}{} & \multicolumn{4}{c|}{ OpenMP } & \multicolumn{4}{c}{ OpenMP } \\
& iterações & 1 & 2 & 4 & iterações & 1 & 2 & 4 \\
\hline 2 & 26 & 79,49 & 54,53 & 52,47 & 20 & 71,90 & 52,32 & 44,24 \\
¿ & 42 & 55,12 & 39,95 & 36,03 & 33 & 37,50 & 29,44 & 26,55 \\
8 & 72 & 29,34 & 25,60 & 25,60 & 39 & 18,37 & 14,76 & 13,60 \\
16 & 82 & 16,96 & 13,37 & 13,70 & 55 & 11,30 & 9,46 & 8,77 \\
\hline
\end{tabular}

Tabela 7. Tempos (seg.) para diferentes estratégias combinatórias - Altix-xe

\begin{tabular}{|c|c|c|c|c|c|c|c|c|c|}
\hline & \multicolumn{4}{|c|}{ Espectral } & \multicolumn{4}{|c|}{ Espectral Valorado + scaling } \\
\hline & & \multirow[b]{2}{*}{ iterações } & \multicolumn{3}{|c|}{ OpenMP } & \multirow[b]{2}{*}{ iterações } & \multicolumn{3}{|c|}{ OpenMP } \\
\hline & & & 1 & 2 & 4 & & 1 & 2 & 4 \\
\hline \multirow{4}{*}{$\bar{\Sigma}$} & 2 & 26 & 45,59 & 34,00 & 26,09 & 20 & 38,88 & 25,73 & 19,85 \\
\hline & 4 & 42 & 31,22 & 22,80 & 26,61 & 33 & 28,46 & 21,57 & 20,67 \\
\hline & 8 & 68 & 17,94 & 13,37 & 13,20 & 39 & 13,19 & 9,32 & 12,84 \\
\hline & 16 & 80 & 9,11 & 6,80 & 6,61 & 56 & 7,68 & 5,36 & 7,35 \\
\hline
\end{tabular}

A Figura 5 apresenta os speedups correspondentes à aplicação das estratégias Espectral Valorado e scaling nos clusters Enterprise 3 e Altix-xe. Apesar dos tempos obtidos pelo cluster Altix-xe serem menores, o cluster Enterprise 3 apresentou melhor escalabilidade, o que evidencia proporcionalmente o tempo de comunicação entre os nós MPI. Este fato ocorre, uma vez que a configuração de cada nó do cluster Altix-xe é superior quando comparado a cada nó do Enterprise 3.

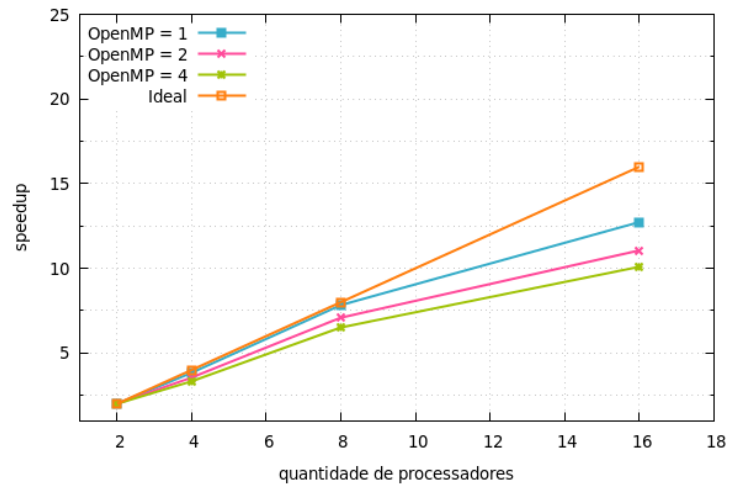

(a) Enterprise 3

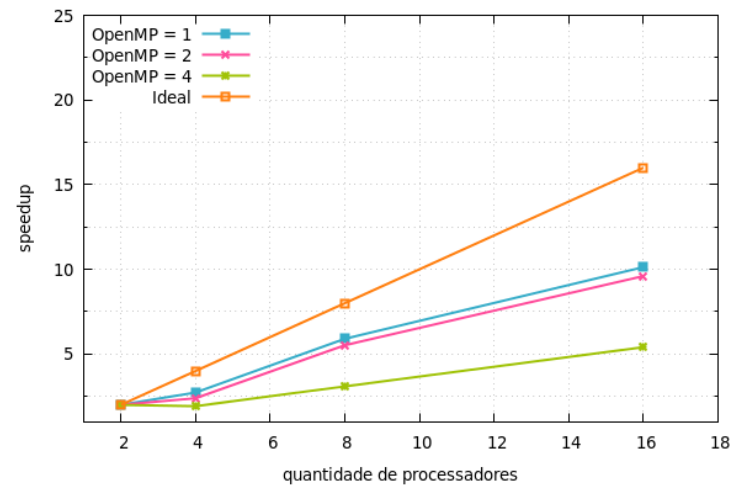

(b) Altix-xe

Figura 5. Speedup: matriz fem_2d_pud

\section{Conclusão e trabalhos futuros}

Nos experimentos realizados, observamos que o precondicionador paralelo híbrido SPIKE depende fortemente das transformações da matriz original através das estratégias combinatórias. Destacamos que nem sempre a aplicação de todas as estratégias simultaneamente conduz a um melhor precondicionador. Entretanto observamos que o precondicionador SPIKE foi eficiente na redução do número de iterações e tempo de processamento do método iterativo GMRES. Como trabalhos futuros, sugerimos implementações paralelas das estratégias combinatórias, visando reduzir o tempo total de processamento e o consumo de memória. 


\section{Agradecimentos}

Os autores agradecem às agências de fomento FAPES, CAPES e CNPq pelo apoio recebido através de bolsas de estudo e suporte financeiro. Agradecemos também ao Laboratório Nacional de Computação Científica (LNCC) pelo uso do cluster Altix-xe.

\section{Referências}

Barnard, S. T., Pothen, A., and Simon, H. D. (1993). A spectral algorithm for envelope reduction of sparse matrices. In Proceedings of the 1993 ACM/IEEE conference on Supercomputing, Supercomputing '93, pages 493-502, New York, NY, USA. ACM.

Davis, T. A. and Hu, Y. (2011). The University of Florida sparse matrix collection. ACM Transactions on Mathematical Software, (1):1:1 - 1:25.

Duff, I. S. and Koster, J. (2000). On algorithms for permuting large entries to the diagonal of a sparse matrix. SIAM J. Matrix Anal. Appl., 22(4):973-996.

Fiedler, M. (1973). Algebraic connectivity of graphs. Czechoslovak Mathematical Journal, 23:298-305.

Hogg, J. and Scott, J. (2015). On the use of suboptimal matchings for scaling and ordering sparse symmetric matrices. Numerical Linear Algebra with Applications.

Manguoglu, M., Koyutürk, M., Sameh, A. H., and Grama, A. (2010). Weighted matrix ordering and parallel banded preconditioners for iterative linear system solvers. SIAM J. Sci. Comput., 32(3):1201-1216.

Manguoglu, M., Sameh, A. H., and Schenk, O. (2009). PSPIKE: A parallel hybrid sparse linear system solver. In Sips, H., Epema, D., and Lin, H.-X., editors, EuroPar 2009 Parallel Processing, volume 5704 of LNCS, pages 797-808. Springer Berlin Heidelberg.

Manne, F. and Sørevik, T. (1995). Optimal partitioning of sequences. J. Algorithms, 19(2):235-249.

Pinar, A. and Aykanat, C. (2004). Fast optimal load balancing algorithms for 1d partitioning. J. Parallel Distrib. Comput., 64(8):974-996.

Polizzi, E. and Sameh, A. H. (2006). A parallel hybrid banded system solver: The SPIKE algorithm. Parallel Comput., 32(2):177-194.

Polizzi, E. and Sameh, A. H. (2007). SPIKE: A parallel environment for solving banded linear systems. Computers \& Fluids, 36(1):113-120.

Sathe, M., Schenk, O., Uçar, B., and Sameh, A. (2012). A Scalable Hybrid Linear Solver Based on Combinatorial Algorithms. In Naumann, U. and Schenk, O., editors, Combinatorial Scientific Computing, Chapman-Hall/CRC Computational Science, pages 95-128. Taylor \& Francis. 